\title{
Age and Growth Determination in Fishes.
}

By Rosa M. Lee.

For $\mathrm{R}$ centuries past it has been known that the scales of fishes showed concentric rings, and later observers have concluded that from the number of these rings the age of the fish could be ascertained.

This discovery was, however, not developed to any great extent until the last ten or twenty years, during which several scientific observers definitely traced the connection between the number of rings on the scales and the number of years of life of certain fishes, the carp amongst freshwater fishes and certain Gadidæ amongst saltwater fishes having been intensively examined and providing a first proof of the correctness of the general theory. The salmon is another fish the economic value and the intrinsic interest of which led to particular attention being paid to its scales in connection with its life-history by many observers, both of professional and amateur status.

But it was the activities of the International Council for the Exploration of the Sea that gave the greatest impetus to the investigation of the age of fishes, and most systematic researches have been carried out with the purpose of correlating the knowledge gained from scale investigations as to the life-history of fishes with that derived from other methods of investigation undertaken by the Council. Amongst the workers of all the nations who have investigated this subject, the Norwegians stand pre-eminent for the very exhaustive researches they have carried on and for the original developments they have made in the methods used, both practical and theoretical. They have concentrated largely on investigating this question in the herring and salmon races, and their contributions to the knowledge of the age distribution and growth of these species have been very valuable.

A great deal of work on the same lines has been carried on by the other countries (including Great Britain) participating in the work of the Council, not only in Europe, but also in America, and the data now collected in regard to various species are extensive enough to enable us to judge the validity of the methods used and the importance of the results obtained.

The theory of age determination, briefly enunciated, is that the periodic quickening and slackening of growth in the fish brought about by the annual changes in the external conditions of their life, viz. summer and winter temperatures, with their accompanying abundance and scarcity of food, or of appetite for food, are reflected in the formation of the scales, and are mainly evident as rings marking the winter growth when development of the scale either slackens or wholly ceases. Such rings can be seen easily with the naked eye or with a low-power lens on the scales of many fishes, and those on the salmon scale illustrated NO. 2654 , VOL. IO6] in Fig. I furnish an example of the general appearance of winter rings.

Such rings are caused by the definite patterns into which the lines on the upper surface of the scale are formed under changing conditions. The arrangement varies for every species of fish, those of the same family being somewhat alike. Indeed, fish can be identified by their scales alone, as has been done on tinned fish purporting to be salmon.

There are striking differences in the appearance of the winter rings in the scales of certain wellknown fishes. In the herring scale the striæ (surface lines) are close and equidistant; they pass from side to side, and appear to be broken by narrow, concentric, transparent rings. In the haddock there are small quadrilaterals arranged concentrically, occurring in zones of wide and narrow growth. In the smelt and the eel this state

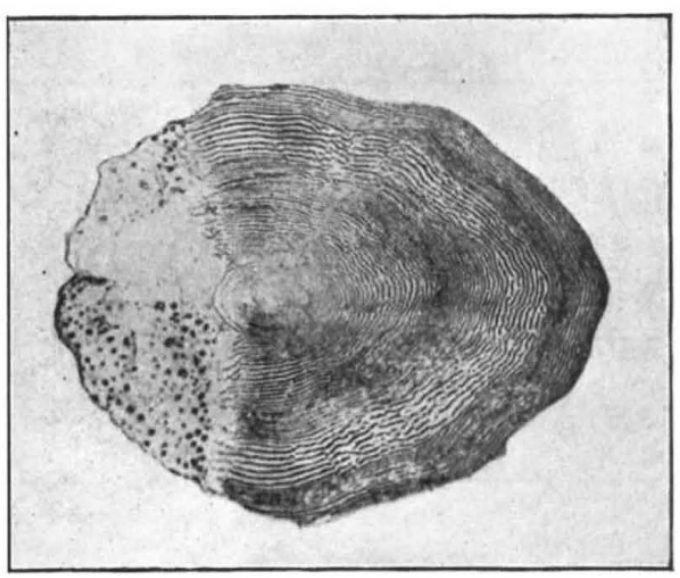

FIG. 1.-Winter rings on a salmon scale.

of wide and narrow zones can be traced, but only with difficulty, and the winter growth is indicated by clear concentric spaces, where the markings are either incomplete or wholly absent. In certain river fish also the winter growth can be traced by the unfinished lines rather than by the narrowing or closing in of the lines, which often marks winter growth.

Underlying all the differences in the patterns on the scales of the various fish a common principle of formation can be traced, and this is undoubtedly due (in normal circumstances) to the annual slackening or cessation of growth associated with winter conditions.

A study of many scales at all times of the year has shown that the discontinuity or incompleteness of the markings (striæ or platelets) may be a temporary characteristic of nearly all stages of growth, and that when growth is active (summer growth) the lines ultimately become complete, but that in the winter they remain incomplete, and 
therefore the discontinuity serves to mark out the winter growth, because the discontinuity becomes permanent, and leaves on the scales the blank spaces or broken lines which are the characteristic winter rings of certain species in addition to the narrowing of the spaces between the lines.

By counting the rings formed on the scale, the age of any individual fish can be ascertained. When large numbers of any one species are examined they can be grouped into "age classes" or "year groups" according to the number of rings. The average size of each group can be determined, and the difference between these sizes gives the first approximation to the amount of annual growth.

This general theory has been confirmed for several species, first by an examination of the growing edge of the scale throughout the season, when the rings have been seen in the course of development, and secondly by marking experiments on the fish (salmon, plaice, cod, etc.), when additional rings corresponding in number to the winters elapsed in the interval between marking and recapture have in every case been seen to be formed on the scales. groups have a probable or natural variation of size, and whether groups of fish of the same ascertained number of rings taken under similar conditions are sufficiently alike in size grouping to give a high probability of being of the same age. These mathematical tests have confirmed the theory that on the average the age determinations of certain species of fish (salmon and herring) are correct up to five or six years of life-that is, those years when they are of most economic importance.

These methods of age determination enabled observers to deduce the approximate growth rates of fish by comparing the sizes of fish of known age. Growth curves so deduced generally rose rapidly from zero for the first two or three years of life, and then the rate of increase fell off rapidly as the fish grew older.

Later researches have demonstrated that this falling off in the growth rate in such fish as herring and plaice and others is not wholly due to a normal slackening in the development, but is made greater by the fact that in several species a segregation according to size takes place, the larger fish of the youngest groups leaving their associates and joining shoais of oider fish, generally in deeper water. Such a segregation has been found to take place at the onset of maturity in certain species. Thus it follows that the youngest year groups in the samples are represented only by the larger individuals of those groups, and their average length is higher than those of the complete year group.

When such selection of size occurs, the average sizes of successive age groups are not strictly comparable with one another.

Fic. 2.-Proportional growth of a fish and its scale.

In practice there are, however, certain modifications and exceptions to the general theory which must be looked for and taken into account. What are known as "false rings" may occur. These bear a resemblance to winter rings, and are probably caused by sudden changes in environment, such as a change of temperature, lack or abundance of food, or even by the fish migrating from one part of the sea to another. These rings can generally be distinguished from true rings in the scales of fishes the habits of which are known. For instance, in salmon there is frequently a grouping of close lines in the middle of the third summer, known as a "summer check."

In very old fish the rings are close together near the edge, and it is difficult to make out their number with exactitude. It may sometimes happen that the latest rings become fused together, and the total number then appears less than those shown on the bony and opercular structures of the same fish.

Results of age determinations of many samples of fish have been studied from the mathematical probability point of view to ascertain whether such NO. 2654 , VOL. IO6]
Norwegian investigators developed the method of ascertaining the growth of individual fish by measuring the comparative distances between the rings on the scales, on the assumption that the scale grows in length in proportion to the length of the fish. Fig. 2 shows a fish of length $\mathrm{L}$ with an enlarged image of its scale. The scale is measured from its centre along the main axis, and also to the edge of the winter rings, giving the values $\mathrm{V}$ (length of scale), $v_{1}, v_{2}$, etc. (lengths from centre to end of first, second winter rings, etc.). If exact proportional growth is assumed between the fish and its scale, it is evident from the parallel lines in the diagram that $l_{1}: v_{1}:: \mathrm{L}: \mathrm{V}$, and the lengths at previous winters $\left(l_{1}, l_{2}\right.$, etc.) are found from the simple formulæ

$$
l_{1}=\frac{v_{1}}{\mathrm{~V}} \cdot \mathrm{L}, \quad l_{2}=\frac{\tau_{2}}{\mathrm{~V}} \cdot \mathrm{L}, \text { etc. }
$$

Such a method enables the data as to growth to be multiplied to a very great extent, and should therefore be most valuable in detecting good and bad years of growth, which can be associated with 
known physical conditions, with the onset of maturity or even with migrations into different waters, and as such they have enormous value.

But, after studying the results obtained, certain investigators have doubted the validity of this method of ascertaining growth. It was noticed that in all species so investigated the calculated lengths for the first and succeeding winters became successively less and less as they were found from older and older fish, and other apparent discrepancies were pointed out by different observers. As a matter of fact, the above method gives only a first approximation to the actual lengths to which the fish grows at the end of each winter of life. It is based on the assumption that the lengths of the scale are themselves proportional to the length of the fish-a closer approximation (but still an approximation), for the calculation is given by taking the increments of growth of the scale as proportional to the increments of growth of the fish, starting from the point at which the scale first appears, which is in most cases an amount sufficiently appreciable to affect the calculations of the first two or three winter lengths considerably.

In Fig. 3 the average relations in the observed lengths of the scale and the corresponding fish, and in the observed lengths of the fish and the corresponding scales, are shown by the dots and crosses respectively.

A mathematical relation can be found by combining all the corresponding measures of scales and fish. This is known as a correlation coefficient, and in the case depicted was very high, viz. $r=0.95$. The points lie very nearly on straight lines, known as "regression lines," the mathematical equations to which are $\mathrm{L}=4 \cdot 8 \mathrm{~V}+3$ and $\mathrm{V}=0.19 \mathrm{~L}-0.36$. From the first of these equations the length of the fish can be calculated when the length of the scale is known from measurements.

The general form of this equation for any series of measurements of fish and their scales is $\mathrm{L}=a \mathrm{~V}+c$, the constant term $c$ corresponding to the length at which the scale begins to grow. In the case of North Sea herring $c$ is about $3 \mathrm{~cm}$.

As in practice only relative lengths of the winter rings are measured on the scale, the constant $a$ in the equation can be eliminated, and the calculated lengths to any previous winter $\left(L_{1}, L_{2}\right.$, etc.) derived from the equation put in the form

$$
\mathrm{L}_{1}=c+\frac{v_{1}}{\mathrm{~V}}(\mathrm{~L}-c), \text { etc. }
$$

The results obtained by the use of this formula

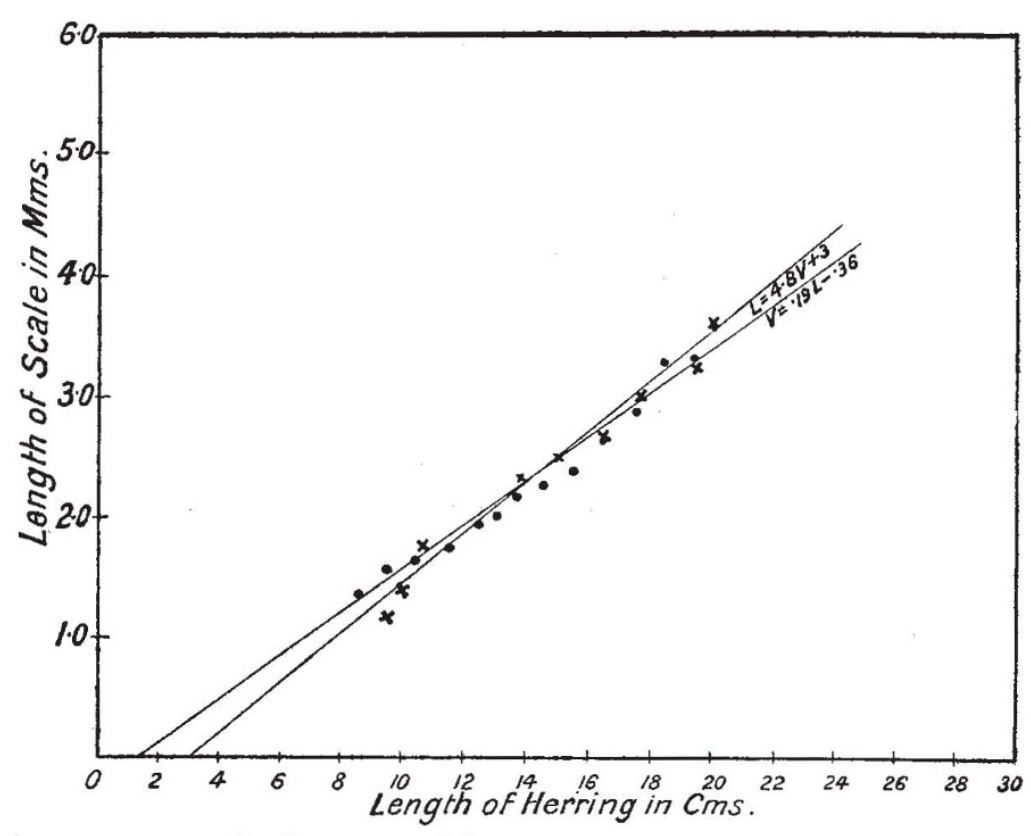

approximate more closely to values derived from observation than in the case of the original formula when $c$ is not taken into account, and the growth measures calculated in this way can therefore be assumed to be a closer approximation to the truth.

In all cases of age and growth determination individual records may be wrong, owing to one or other of many difficulties that may occur in deciphering the scale, but the latest work on the subject has shown that average values, both of age distribution in a sample and of amount of growth, are approximately correct.

\section{The Structure of the Atom.}

By C. G. Darwin.

\section{I.--Atomic Number.}

THE study of the interior structure of the atom was initiated about twenty-five years ago by J. J. Thomson's discovery of the electron. Electrons are particles of negative electricity of charge $4.77 \times 10^{-10}$ electrostatic units and mass $9.0 \times 10^{-28} \mathrm{gr}$., and they were shown to be conNo. 2654 , VOL. IO67 stituents of every type of matter. Some years previously Thomson had shown on theoretical grounds that any charged body should possess extra mass on account of its charge, and these two facts taken together suggested the possibility of an electrical theory of matter, whereby all mass should be electromagnetic in origin, and 